\title{
Estimating Contact Forces and Moments for Walking Robots and Exoskeletons using Complementary Energy Methods
}

\author{
Jonas Vantilt ${ }^{1}$, Chetan Giraddi ${ }^{1}$, Erwin Aertbeliën ${ }^{1}$, Friedl De Groote ${ }^{2}$ and Joris De Schutter ${ }^{1}$
}

\begin{abstract}
When walking robots and exoskeletons make multiple independent contacts, the inverse dynamics problem requires additional knowledge about the contact forces and moments. To avoid measuring the contact forces and moments, many inverse dynamics controllers for walking robots optimize an objective like minimizing torques or contact forces. In order to get a solution closer to the real solution, the underlying physical principles need to be included. This is achieved by relying on the minimization of complementary energy, which is a well known method in structural engineering. The proposed method relies on physical properties (stiffness) to obtain the additional knowledge to solve the contact forces and moments. In addition, it has the same form as the methods used in the literature. The validation on a bilateral lower-limb exoskeleton shows that the proposed method is able to predict the contact forces and moments sufficiently well, while being robust against modelling errors. Modelling the dominating flexibilities suffices to achieve adequate results, making this method especially interesting for series elastic actuated robots.
\end{abstract}

Index Terms-Dynamics, Flexible Robots, Wearable Robots, Legged Robots

\section{INTRODUCTION}

$\mathbf{M}$ ANY model-based control methodologies for walking humanoid robots or lower-limb (assistive) exoskeletons require the evaluation of an inverse dynamics model. In humanoids, torques need to be computed to follow planned motion or interaction with the environment. In a lower-limb assistive exoskeleton, torques need to be computed to follow the human operator in a transparent way while additional assistive torques are applied. However, multiple over-constrained contacts with the environment are present underneath the feet during overground walking and other activities. When this happens, the dynamic equations alone are not sufficient to solve the inverse dynamics. Additional knowledge about the contact forces and moments is required. We refer to the combination of forces and moments at each contact as wrench from here on.

Manuscript received: February, 23, 2018; Revised April, 14, 2018; Accepted June, 17, 2018.

This paper was recommended for publication by Editor Nikos Tsagarakis upon evaluation of the Associate Editor and Reviewers' comments. This work was supported by the Agency Flanders Innovation \& Entrepreneurship (VLAIO)

${ }^{1}$ The authors are with the Robotics Research Group, Department of Mechanical Engineering, KU Leuven, 3000 Leuven, Belgium and are members of Flanders Make firstname. lastname@kuleuven.be

${ }^{2}$ Friedl De Groote is with the Department of Kinesiology, KU Leuven, 3000 Leuven, Belgium friedl. degroote@kuleuven. be

Digital Object Identifier (DOI): see top of this page.
Force cells underneath the feet can measure these contact wrenches [1][2], but in many use cases direct measurement has significant disadvantages. First, contact can occur at locations without force cell, resulting in incomplete contact force registration. Second, expensive force cells increase the cost of the application. Third, force cells are susceptible to overloading and can make the device less robust and less deployable in real-world applications. Due to space requirements, these problems are even worse for exoskeletons than for humanoid robots. Therefore, there is a lot of interest in computing these contact wrenches with other methods and thus avoiding the need for direct measurements.

A typical way to avoid direct force measurements is to solve the over-constrained inverse dynamics problem by optimizing an objective function. In a first category of approaches, the dynamics are projected onto the constrained dynamics by taking the dynamically consistent generalized inverse of the contact Jacobian [3][4][5]. The weights of these pseudo-inverses rely on the mass matrix of the robot, which is computationally expensive. Mistry et al. [6] reduce the computational load by using a QR decomposition of the contact Jacobian to project the dynamics onto the constrained dynamics. Righetti et al. [7] show that different methods [3][4][6] provide the same solution of the inverse dynamics. In their follow-up work [8], they show that most existing methods can be formulated as a minimization of linear and quadratic costs on the contact wrenches and control torques. This optimization solves the inverse dynamics when over-constrained contacts with the environment are present.

The work of Righetti et al. [8] solves the inverse dynamics as a minimization of linear and quadratic costs on contact wrenches and control torques:

$$
\min \frac{1}{2} \boldsymbol{\tau}^{T} \mathbf{W}_{\boldsymbol{\tau}} \boldsymbol{\tau}+\boldsymbol{b}_{\tau}^{T} \boldsymbol{\tau}+\frac{1}{2} \boldsymbol{w}_{c}^{T} \mathbf{W}_{\boldsymbol{w}} \boldsymbol{w}_{c}+\boldsymbol{b}_{\boldsymbol{w}}^{T} \boldsymbol{w}_{c}
$$

with $\mathbf{W}_{\boldsymbol{\tau}}$ and $\mathbf{W}_{\boldsymbol{w}}$ the (assumed positive definite) weighting matrices of the control torques and contact wrenches, respectively. The vectors $\boldsymbol{b}_{\tau}$ and $\boldsymbol{b}_{\boldsymbol{w}}$ represent the linear factor for the control torques and contact wrenches, respectively. One aspect not solved in their work is how to find the weighting matrices and linear factors. In order to get a stable solution, tuning is required for every new situation. As will be clear from this paper, our proposed method will result in a final expression similar to eq. (1). However, our method minimizes complementary energy, while the literature minimizes a weighted combination of control torques and contact wrenches. As a 
result, the major difference is that our method directly gives the weighting matrices and linear factors $\boldsymbol{b}$ for every situation and (extensive) tuning is no longer required.

A second category of approaches similar to the methods above computes the over-constrained inverse dynamics by formulating an optimization problem in the following variables: the joint accelerations $\ddot{\boldsymbol{q}}$, the actuation torques $\boldsymbol{\tau}$ and the contact wrenches $\boldsymbol{w}$. Hence, the contact wrenches are explicitly part of the optimization. Any constraint resulting from a contact with the environment (e.g. stay in friction cone) can then be formulated in these variables. The computed actuation torques and contact wenches are a result of a tradeoff between all these constraints [9][10][11]. The bottom line of all these methods is that the equations of motion are insufficient to solve the inverse dynamics with multiple contacts. In the literature, this is solved by optimizing some objective function.

Another way to avoid measuring the contact wrenches is to rely on Disturbance Observers (DOB) or Reaction Force observers (RFOB) [12]. A non-linear DOB [13] is required due to the coupling between the joints. Although these methods have been applied to improve assistance delivered to a person in some upper-limb exoskeletons [14][15], DOBs are not yet used in humanoid robots to estimate or control ground contact wrenches. These DOBs have several disadvantages. First, they always introduce some time delay due to the filtering and they show a transient behaviour. Second, although methods to develop non-linear DOBs [13][14] exist, they are not guaranteed to provide stable results. Third, when closing the loop with DOBs, the stability of the system can degrade, which requires a further reduction of DOB bandwidth. Fourth, a DOB is unable to distinguish between the contact wrenches and other disturbances introduced by the task or person. This last disadvantage is the most important argument to estimate contact wrenches with another method and use a DOB for estimating/rejecting the disturbances which are too hard to model.

Solving the inverse dynamics of over-constrained multiple contacts has a large similarity with hyperstatic structures in structural engineering. In these hyperstatic situations, the force and moment equilibrium equations are insufficient to solve all contact forces and moments. Hence, additional equations are required. These additional equations come from the constitutional laws (stiffness relations) and the compatibility between displacement and strain [16][17]. The previously mentioned approaches in the robotics literature do not use this stiffness information and the compatibility between displacement and strain. Complementary energy is a method in structural engineering to obtain the additional compatibility equations to solve the hyperstatic situation.

The main contribution of this paper is the introduction of the complementary energy method in robotics to solve contact forces and moments in inverse dynamics controllers of robots. First, the complementary energy method is expanded to dynamic systems using d'Alembert's principle of inertial forces. Second, the proposed method is compared against the existing optimizations [8]. The advantage of the proposed method is the ability to directly derive the weighting matrices of the optimization by minimizing the complementary energy. Third, the method is validated on a lower-limb exoskeleton without person.

Section II starts with the floating-base formulation and the formulation of the complementary energy. In section III the method is applied to the exoskeleton at hand and the complementary energy of the exoskeleton is derived. Section IV describes the experiments and results and is followed by the discussion and the conclusions.

\section{COMPLEMENTARY ENERGY METHOD}

\section{A. Floating-base dynamics}

The floating-base model [18] of a robot in contact with the environment is represented by the following $r$ equations:

$$
\mathbf{M}(\boldsymbol{q}) \ddot{\boldsymbol{q}}+\mathbf{C}(\boldsymbol{q}, \dot{\boldsymbol{q}}) \dot{\boldsymbol{q}}+\mathbf{G}(\boldsymbol{q})=\mathbf{S}^{T} \boldsymbol{\tau}+\mathbf{J}_{c}^{T}(\boldsymbol{q}) \boldsymbol{w}_{c}
$$

with the assumption that no energy is dissipated in the ground contacts (e.g. by sliding friction) and with the following definitions:

- $\boldsymbol{q}, \dot{\boldsymbol{q}}, \ddot{\boldsymbol{q}} \in \mathbb{R}^{r}$ : the state vector, consisting of six floatingbase variables and $n$ joint angles $\phi_{i}$,

- $\mathbf{M} \in \mathbb{R}^{r \times r}$ : the inertia matrix,

- $\mathbf{C} \in \mathbb{R}^{r \times r}$ : the Coriolis and centrifugal matrix,

- $\mathbf{G} \in \mathbb{R}^{r}$ : the gravitational vector,

- $\boldsymbol{\tau} \in \mathbb{R}^{n}$ : the actuation torques,

- $\mathbf{S}=\left[\begin{array}{ll}\mathbf{0}_{n \times 6} & \mathbf{I}_{n \times n}\end{array}\right]$ : the actuation selection matrix,

- $\boldsymbol{w}_{c} \in \mathbb{R}^{k}$ : the vector of all contact forces/moments,

- $\mathbf{J}_{c} \in \mathbb{R}^{k \times r}$ : the contact/constraint Jacobian.

From here on, the dependence of matrices and vectors on $\boldsymbol{q}$ or $\dot{q}$ is omitted in the equations. The number of actuated joints of the robot is $n ; r=n+6$ is the number of floating-base model states and $k$ is the dimension of the independent contact constraints. For example, with one non-sliding foot flat on the ground $k=6$; with two non-sliding feet flat on the ground $k=12$. The kinematics at the contact points are constrained using the additional equations:

$$
\begin{array}{r}
\dot{\boldsymbol{p}}_{c}=\mathbf{J}_{c} \dot{\boldsymbol{q}}=0, \\
\ddot{\boldsymbol{p}}_{c}=\mathbf{J}_{c} \ddot{\boldsymbol{q}}+\dot{\mathbf{J}}_{c} \dot{\boldsymbol{q}}=\mathbf{J}_{c} \ddot{\boldsymbol{q}}+\left(\sum_{j=1}^{r} \frac{\partial \mathbf{J}_{c}}{\partial q_{j}} \dot{q}_{j}\right) \dot{\boldsymbol{q}}=0,
\end{array}
$$

with $\boldsymbol{p}_{c}$ representing the positions and orientation of the contact(s) with respect to the world frame.

In the inverse dynamics problem, the kinematics are known $(\boldsymbol{q}, \dot{\boldsymbol{q}}, \ddot{\boldsymbol{q}})$, but the force and moment variables are unknown $\left(\boldsymbol{\tau}, \boldsymbol{w}_{c}\right)$. Therefore, we have $r=6+n$ equations for $n+k$ variables. We consider here the over-constrained case $(k>6)$ where the number of independent contacts exceeds the number of floating-base coordinates. The equations are then no longer sufficient to solve for all the joint torques $\boldsymbol{\tau}$ and contact wrenches $\boldsymbol{w}_{c}$. Additional knowledge on the $k$ constraint wrenches in $\boldsymbol{w}_{c}$ is required.

These additional equations can come from minimizing a cost function of actuation torques and/or constraint wrenches, as is done by Righetti et al. [8]. However, we aim to find a physics-based solution instead of a regularizing solution. Minimizing the complementary energy is directly related to 
physical compatibility in displacement and deformations [16] of the robot. In contrast, minimizing a cost function of actuation torques and constraint wrenches [8] has no physical relation with the robot.

\section{B. Formulation of complementary energy}

In structural engineering, when considering hyperstatic structures, a complete set of equations is obtained by considering three types of equations: the equilibrium equations, the constitutive laws, and the compatibility equations [16]. The well-known principle of d'Alembert [19] allows us to treat dynamics as external forces. Concretely, the left-hand side of (2) is grouped into a vector variable $\tau_{d y n}$, which acts in the same way as external forces and moments on a static system. In this way, this robot dynamic problem is transformed into a static problem where structural engineering methods can be applied.

The constitutive laws describe the relation between stress and strain by involving the material or substance of the structure. Here, they consist of the stiffness relations for elements inside the robotic structure. All stiffness relations can be considered, describing the compliance of the links, joints and contacts. When constitutive equations are introduced, also additional compatibility equations are needed, i.e. equations that ensure that the movement due to the structural compliance is compatible with the mechanism. To establish these compatibility equations, structural engineers have developed complementary energy methods [16]. Complementary energy is an entity similar to energy, but expressed in terms of forces and moments instead of displacements. The minimization of this complementary energy allows us to solve the equilibrium equations without having to explicitly derive all the, possibly complex, compatibility relations.

Internal moments and forces in a compliant element produce the internal complementary energy, such as the deflection force of a translational spring. The contribution to complementary energy from the unknown external contact wrenches remains zero under the assumption of non-sliding contact. The same assumption was already made when establishing (2). The external complementary energy only depends on the known/desired dynamics, which acts as an external force thanks to principle of d'Alembert. This external complementary energy is thus independent of $\boldsymbol{\tau}$ and $\boldsymbol{w}_{c}$ and therefore can be left out of the complementary energy minimization. For a given kinematic state $(\boldsymbol{q}, \dot{\boldsymbol{q}}$ and $\ddot{\boldsymbol{q}})$, all internal forces and moments depend affinely on $\boldsymbol{\tau}$ and $\boldsymbol{w}_{c}$. This can be seen by considering the (linear) equilibrium equations based on the free body diagram of appropriately isolated parts of the robotic structure. Thus, the minimization of the complementary energy depends on $\tau$ and $\boldsymbol{w}_{c}$ :

$$
\begin{aligned}
& \min _{\boldsymbol{w}_{c}, \boldsymbol{\tau}} A^{*}\left(\boldsymbol{w}_{c}, \boldsymbol{\tau}\right), \\
& \text { s.t. } \boldsymbol{\tau}_{d y n}=\mathbf{S}^{T} \boldsymbol{\tau}+\mathbf{J}_{c}^{T}(\boldsymbol{q}) \boldsymbol{w}_{c},
\end{aligned}
$$

where $\boldsymbol{\tau}_{d y n}$ is known/desired and $A^{*}$ is the complementary energy. The star emphasises the complementary nature. This minimization has to be solved each time instance.

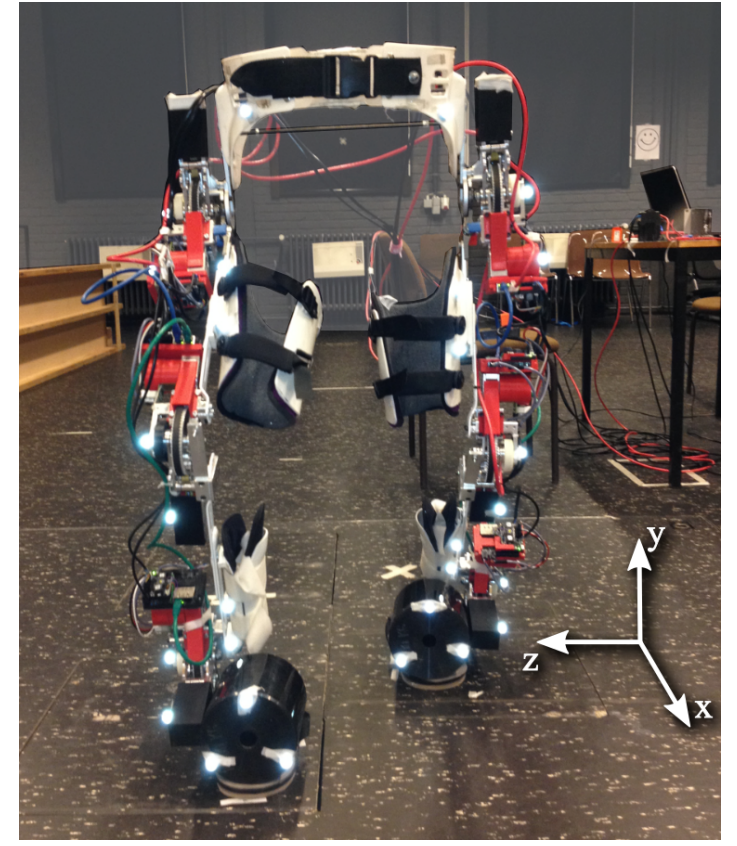

Fig. 1. The exoskeleton with SEAs used to validate the method to estimate the contact forces. The world reference frame is located in the right lower corner in white

Modelling the complementary energy of all stiffness elements and internal forces and moments in a robot is a tedious task. However, this effort can be reduced when having a closer look at the energy expression of a linear spring:

$$
A^{*}\left(F_{s p r}\right)=\int_{0}^{F_{s p r}} u(F) d F=\frac{F_{s p r}^{2}}{2 K},
$$

with $F_{s p r}=K u$ the spring force, $u$ the deformation and $K$ the stiffness. All complementary energy terms have a similar formulation: a force or moment squared divided by the stiffness. For equal forces, the energy changes inversely proportional to the stiffness. This implies that when stiffness gets ten times larger, the energy decreases by a factor of ten. In addition, from the minimization of the complementary energy in eq. (5) together with the energy of a spring eq. (7), it is clear the stiffness operates as weighting of the forces and moments. Hence, the magnitude of the stiffness of the elements does not matter, only their mutual relative magnitudes. When considering a kinematic chain of links and joints that connects the floating base to the environment, very stiff elements of the chain contribute less to the total complementary energy than less stiff elements of the same chain. As a guideline to simplify the stiffness models, elements with a stiffness more than one order of magnitude larger than the other elements can be excluded from the energy. For example, in a structure with stiff links and compliant joints, there is no need to model the stiff links.

Because the complementary energy terms all have the form of a force term squared divided by stiffness, the complementary energy can be written in the same form as eq. (1). Hence, it can be written as the solution that Righetti et al. [8] found to be a generalization of existing inverse dynamics solvers in the literature. However, our method directly provides the 


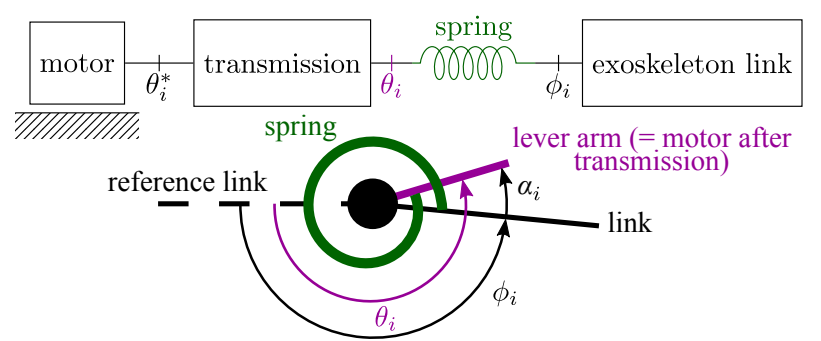

Fig. 2. Model of the SEA. The SEA consists of a motor, transmission, spring and outgoing link, as seen in the top figure. The SEA is modelled as a rotary spring where the torque depends on the spring angle $\alpha_{i}$.
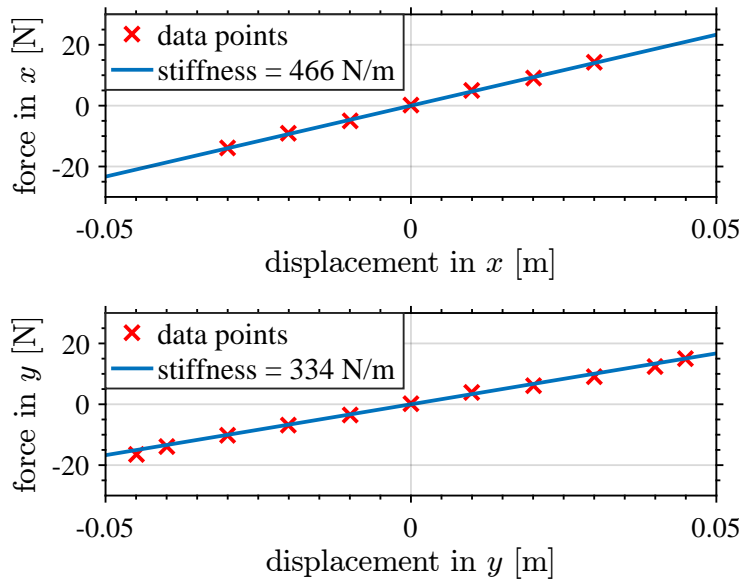

Fig. 3. The stiffness of the pelvis is approximated by a linear stiffness relation between deflection and force. The stiffness in the $x$ and $y$ direction are shown in the top graph and bottom graph, respectively.

weighing matrices and linear factor $(\boldsymbol{b})$ and relates them to the stiffness of the robot. No tuning of weighing matrices or $\boldsymbol{b}$ is required, because they are directly following from the complementary energy. The weighing matrix contains the inverse of the modelled stiffness of the robot. In case of linear stiffness, the weighing matrix is constant and positive semidefinite, making the optimization quadratic and convex. In case of non-linear stiffness, the weighing matrix depends on the actuation torques and contact wrenches and the optimization becomes non-linear.

\section{APPLICATION TO AN EXOSKELETON}

The purpose of this section is twofold: firstly, it discusses how to implement the above method in a practical set-up; secondly, it validates our method on a lower-limb exoskeleton with Series Elastic Actuators (SEAs). This exoskeleton was developed during the MIRAD project [20], and is shown in Fig. 1. The exoskeleton design contains six actuators $(n=6)$ for hip, knee and ankle joint, moving in the sagittal plane, for both legs. As a result, a 2D model is sufficient for this exoskeleton. This reduces the number of floating-base state variables and equations to three, while $k=6$ when two non-sliding feet are on the ground. The contact wrenches, consisting of six forces and moments, are assembled $\boldsymbol{w}_{c}$ :

$$
\boldsymbol{w}_{c}=\left[\begin{array}{llllll}
F_{x, L} & F_{y, L} & M_{z, L} & F_{x, R} & F_{y, R} & M_{z, R}
\end{array}\right]^{T},
$$

TABLE I

THE LINEARISED JOINT SPRING STIFFNESS

\begin{tabular}{c|c|c|c|c|c|c} 
joint & hip $_{L}$ & knee $_{L}$ & ankle $_{L}$ & hip $_{R}$ & knee $_{R}$ & ankle $_{R}$ \\
\hline$K\left[\frac{N m}{r a d}\right]$ & 35.4 & 33.4 & 33.7 & 33.2 & 36.2 & 37.6
\end{tabular}

with the heel as reference point. The subscripts $L$ and $R$ refer to the left and right foot, and the subscripts $x, y$ and $z$ refer to the frame as indicated in Fig. 1.

The exoskeleton has SEAs and the dynamic model is described by combining the floating base model [18] and the flexible joint model from the Langrangian of the whole robot [21] with large transmission ratios (our exoskeleton has a transmission ratio of 188$)$ :

$$
\begin{aligned}
\mathbf{M}(\boldsymbol{q}) \ddot{\boldsymbol{q}}+\mathbf{C}(\boldsymbol{q}, \dot{\boldsymbol{q}}) \dot{\boldsymbol{q}}+\mathbf{G}(\boldsymbol{q}) & =\mathbf{S}^{T} \boldsymbol{\tau}+\mathbf{J}_{c}^{T}(\boldsymbol{q}) \boldsymbol{w}_{c} \\
\mathbf{B} \ddot{\boldsymbol{\theta}}+\boldsymbol{\tau} & =\boldsymbol{\tau}_{\text {motor }}
\end{aligned}
$$

where (9) is the same as (2) and with $\boldsymbol{\theta}$ the vector of motor angles after transmission. The motor torques $\boldsymbol{\tau}_{\text {motor }}$ are used to accelerate the motors with the (diagonal) inertia matrix $\mathbf{B}$ and to generate the actuation torques $\boldsymbol{\tau}$. These actuation torques are the spring torques. Any friction in the joints or gears can be included with a friction term $\tau_{\text {fric }}$ in the left hand side of (9) and (10), respectively.

The SEAs are modelled as rotary springs as shown in Fig. 2. The spring deflection angle $\alpha_{i}$ is the difference in motor and joint angle: $\theta_{i}-\phi_{i}$. The torque angle relationship of each spring is identified in [22] and is given by:

$$
\tau_{i}=f_{i}\left(\alpha_{i}\right)
$$

with $f_{i}$ a non-linear torque-angle relation. The complementary energy $A_{s p r}^{*}$ in the $n$ actuator springs then becomes:

$$
A_{s p r}^{*}(\boldsymbol{\tau})=\sum_{i=1}^{n} \int_{0}^{\tau_{i}} f_{i}^{-1}\left(t_{i}\right) d t_{i}
$$

with $t_{i}$ representing the integration variable for torque. Our proposed method has an advantage when working with SEA robots or when the flexibility of the robot is predominantly present in the actuator. In our exoskeleton, the frame is significantly stiffer than the SEA springs. Hence, the high stiffness and corresponding complementary energy of the frame can be ignored compared to the springs.

All the links of the MIRAD exoskeleton have a high stiffness compared to the joints, except for the pelvis link. As a result, the complementary energy of the pelvis link cannot be neglected. The complementary energy of the pelvis link depends on the ground reaction wrenches $\boldsymbol{w}_{c}$ and the dynamics (2). Because the exact stiffness properties of the pelvis are unknown, they are approximated using experimental force-deflection data of the pelvis in Fig. 3. The pelvis was clamped on one side and both the deflection and force were measured on the free side. A linearised stiffness is derived 
for the pelvis for both the force in $x$ and $y$, resulting in a complementary energy $A_{\text {pelv }}^{*}$ :

$$
A_{\text {pelv }}^{*}\left(\boldsymbol{w}_{c}\right)=\frac{F_{x, \text { pelv }}^{2}}{2 K_{x, \text { pelv }}}+\frac{F_{y, \text { pelv }}^{2}}{2 K_{y, p e l v}}
$$

with $F_{x, p e l v}$ and $F_{y, p e l v}$ the force transmitted through the pelvis in the $x$ and $y$ direction, respectively. The $F_{x, p e l v}$ force is the difference in horizontal force through the left and right leg. For the left leg this becomes:

$$
F_{x, p e l v, L}=F_{x, L}-\sum_{j} m_{j, L} a_{x, j, L}
$$

with $a$ the acceleration and $m$ the mass of each link $j$ (foot, shank and thigh) of the left leg $L$. This results in the force transmitted through pelvis in $x$ :

$$
F_{x, p e l v}=F_{x, L}-F_{x, R}-\sum_{j} m_{j, L} a_{x, j, L}+\sum_{j} m_{j, R} a_{x, j, R}
$$

The complementary internal energy of the exoskeleton with SEAs is then obtained by combining (12) and (13):

$$
A^{*}\left(\boldsymbol{\tau}, \boldsymbol{w}_{c}\right)=\sum_{i=1}^{n} \int_{0}^{\tau_{i}} f_{i}^{-1}\left(t_{i}\right) d t_{i}+\frac{F_{x, p e l v}^{2}}{2 K_{x, p e l v}}+\frac{F_{y, p e l v}^{2}}{2 K_{y, p e l v}},
$$

which is simplified in the case of linear(ised) springs:

$$
A^{*}\left(\boldsymbol{\tau}, \boldsymbol{w}_{c}\right)=\sum_{i=1}^{n} \frac{\tau_{i}^{2}}{2 K_{i}}+\frac{F_{x, \text { pelv }}^{2}}{2 K_{x, \text { pelv }}}+\frac{F_{y, \text { pelv }}^{2}}{2 K_{y, \text { pelv }}},
$$

with $K_{i}=d f_{i}\left(\alpha_{i}\right) / d \alpha_{i}$ the linear(ised) stiffness of the spring. The linearised spring stiffness of each joint is given in Table I.

A model for the internal complementary energy in the robot system is now established and the methodology of section II can be applied by solving the minimization problem in (5) and (6). The complementary energy of the non-linear springs in eq. (16) is used in eq. (5).

\section{EXPERIMENTS AND RESULTS}

To validate the proposed methodology for ground force estimation under dynamic conditions, the MIRAD exoskeleton was put in a non-symmetric configuration on two force plates, see Fig. 1. The average reference exoskeleton joint angles $\phi_{i}$ were: $\left[\begin{array}{llllll}66 & -56 & 88 & 53 & -80 & 125\end{array}\right][\mathrm{deg}]$ for the hip, knee and ankle of the left and right leg, respectively. A PD position controller for each of the six joints imposed a periodic motion to excite the dynamics of the exoskeleton. The reference trajectories of the joints are given in Fig. 4. The transient behaviour is not shown as they are not relevant for the message of the paper. The motion was initialized by first taking the amplitude of the periodic trajectories to zero and then gradually increasing the amplitude. This made sure transients would not destabilise the simple PD controller. To make sure the ground reaction wrenches and dynamics are not influenced by any estimation, the PD position controller did not use any estimation. Hence, the proposed estimation and other methods from literature were applied on the measured data to estimate the ground reaction wrenches off-line.

Weights were put on each exoskeleton foot to prevent the foot from loosing ground contact and allowing more dynamic motions of the exoskeleton. The mass of the weights and

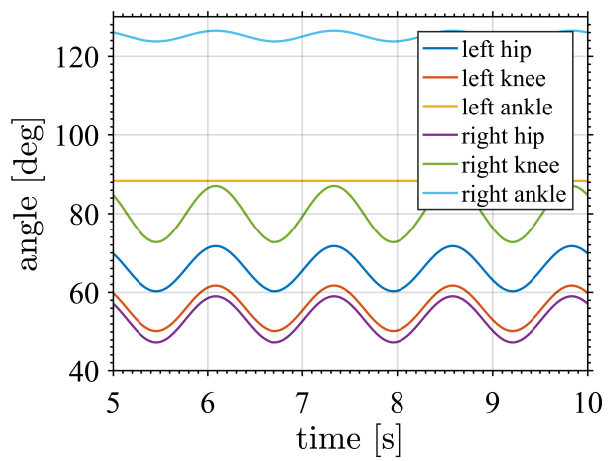

Fig. 4. The absolute joint trajectories of the exoskeleton in degrees. The actual knee angles are negative. When the exoskeleton is standing upright, hip and knee angles are zero and the ankles are 90 degrees.

TABLE II

RMS JOINT TORQUE ERROR OF THE ESTIMATION BASED ON THE COMPLEMENTARY ENERGY USING THE NON-LINEAR STIFFNESS AND THE LINEARISED STIFFNESS

\begin{tabular}{c|c|c} 
joint torque & non-lin. stiffness eq. (16) & lin. stiffness eq. (17) \\
\hline$\tau_{L, \text { hip }}$ & $0.57 \mathrm{Nm}$ & $0.59 \mathrm{Nm}$ \\
$\tau_{L, \text { knee }}$ & $0.80 \mathrm{Nm}$ & $0.93 \mathrm{Nm}$ \\
$\tau_{L, \text { ankle }}$ & $0.53 \mathrm{Nm}$ & $0.77 \mathrm{Nm}$ \\
$\tau_{R, \text { hip }}$ & $0.42 \mathrm{Nm}$ & $0.45 \mathrm{Nm}$ \\
$\tau_{R, \text { knee }}$ & $0.56 \mathrm{Nm}$ & $0.75 \mathrm{Nm}$ \\
$\tau_{R, \text { ankle }}$ & $0.52 \mathrm{Nm}$ & $0.70 \mathrm{Nm}$
\end{tabular}

their location with respect to the force plates are known. The wrenches caused by the weights were subtracted from the measured force plate wrenches to obtain the measured force plate wrenches of the exoskeleton alone. The feet and the weights did not move with respect to the ground. Hence, these added weights did not influence the proposed method to estimate the contact forces and moments in any way.

The experiments were performed in a motion analysis lab. The exoskeleton was placed on force plates (AMTI, Waterworn, USA) to measure the ground reaction forces and moments at a sample frequency of $1000 \mathrm{~Hz}$. The location of the feet with respect to the force plates was measured using a reflective marker motion capture system consisting of ten cameras (Vicon Motion Systems, Oxford Metrics, UK) sampled at $100 \mathrm{~Hz}$. The joint angles $\phi_{i}$ and motor angles after transmission $\theta_{i}$ are all measured by encoders on the exoskeleton and the dynamic model and parameters of the exoskeleton were known [22].

The estimated ground reaction wrenches (in red) are resembling the measured ones (in blue) in Fig. 5. The small errors still remaining are partially caused by errors in the dynamic model, measurement and filtering errors. The green curves show the ground reaction force estimations using the method described in [6], which was used as benchmark for comparison with the state of the art. In this method, the norm of the torques were minimized with a unit weight on all actuated joints. Hence, no tuning was performed. These benchmark estimations differ significantly from the measured reaction forces. The errors between the measurements and benchmark 


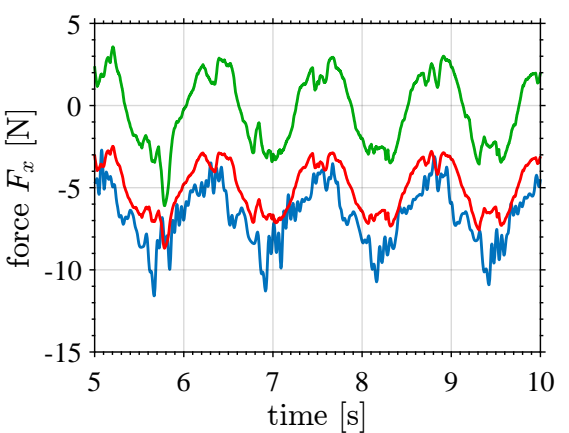

(a) $F_{x}$ left foot

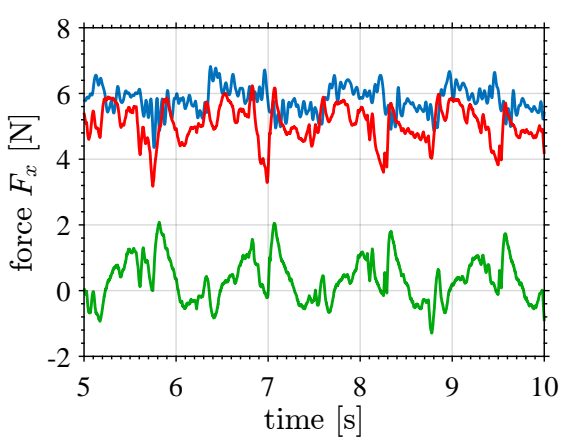

(d) $F_{x}$ right foot

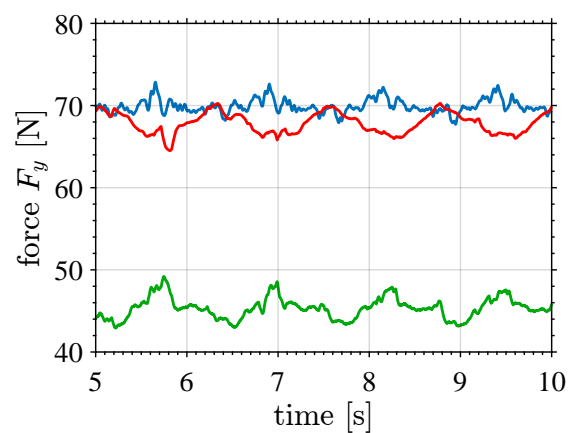

(b) $F_{y}$ left foot

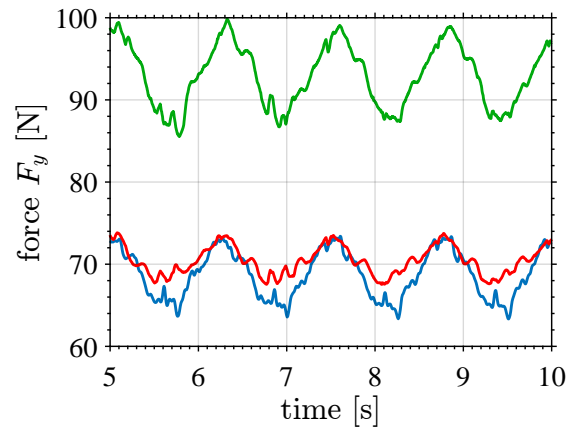

(e) $F_{y}$ right foot

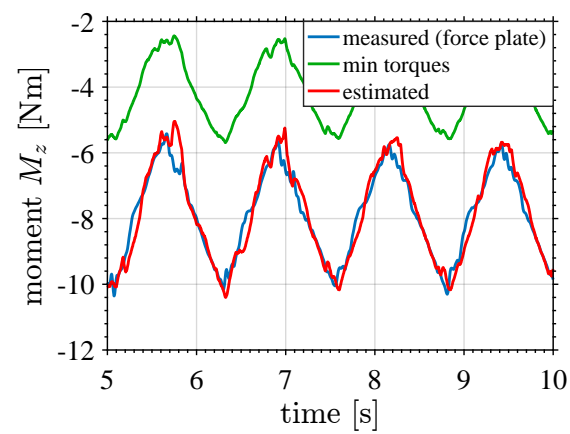

(c) $M_{z}$ left foot

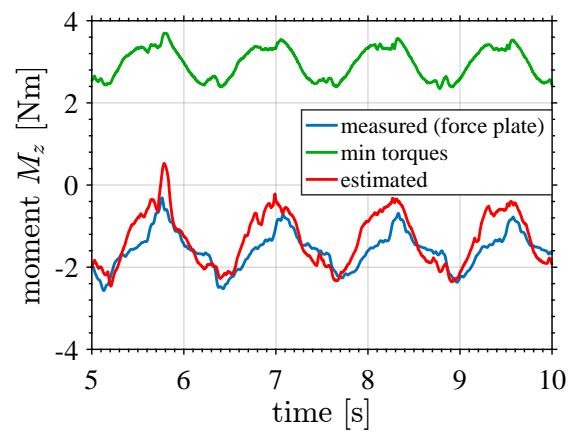

(f) $M_{z}$ right foot

Fig. 5. The estimated ground reaction wrenches in red are compared against the measured ones in blue and the solution of a benchmark method, which minimizes the norm of actuation torques, in green. The top three and lower three figures represent the moment, force in $x$ and $y$ under the heel of the left foot and the right foot, respectively.

estimation are around $5 \mathrm{Nm}$ for the moment, $5 \mathrm{~N}$ for the $x$ force and more than $20 \mathrm{~N}$ error for the $y$ force.

We also assessed the accuracy of the joint torques computed based on the estimated contact wrenches in Fig. 6. As the dynamic model is the same in each method, the difference in the torques is caused by the difference in the ground reaction wrenches. The joint torques are similar when using the measured (in blue) and estimated contact wrenches (in red). Although the hip joints curves are not really similar, the order of magnitude is similar. The errors in the hip joints are caused by subtracting large hip torques from the ground reaction wrenches $\mathbf{J}_{c, h i p}^{T}(\boldsymbol{q}) \boldsymbol{w}_{c}$ from large floating base hip torques $\boldsymbol{\tau}_{\text {dyn,hip. }}$. The contact wrenches from the benchmark method [6] (in green) result in large errors compared to the torques from the measured contact wrenches. These errors are not only biases, as the amplitude is also different.

Table II shows the Root Mean Square (RMS) error of the joint torques using the estimated contact wrench in the left column. This is the error between the estimated (red) and measured (blue) joint torques in Fig. 6. In addition, the proposed energy method is also calculated using linearised joint stiffness. The solution of the linearised springs is also considered as it reduces the complexity of the modelling and the computation time. These results are not shown in Fig. 5 and Fig. 6 to not overload the figures. The RMS error between the linearised joint torque estimation and the measured joint torque is given in the right column of Table II. The estimation based on linearised stiffness has slightly larger errors than the one based on the non-linear stiffness.

\section{DISCUSSION}

The proposed method is able to accurately estimate the ground reaction wrenches, see Fig. 5. The benchmark method that minimizes joint torques displays large errors compared to the measured forces and moments. This originates from the fact the benchmark only minimizes the norm of the joint torques with equal weights for all joints. The deformation and hence energy in the pelvis is not accounted for in the benchmark method. Moreover, it is not possible to take this pelvis link effect into account in a straightforward way in the benchmark method. The proposed method takes the stiffness and deformation of the robot into account. This results in solutions close to the measured contact forces and moments.

The small errors between the estimated and measured contact wrenches have only a small effect on the resulting calculated joint torques, see Fig. 6. The estimated force in $y$ deviates $5 \mathrm{~N}$ at its peaks in Fig. $5 \mathrm{~b}$ and Fig. $5 \mathrm{e}$ and the moment at the right foot differs at most $0.5 \mathrm{Nm}$ in Fig. $5 \mathrm{f}$. This results in joint torque errors of less than $0.5 \mathrm{Nm}$ as is seen in Fig. 6. Again, the solution of the benchmark joint torques differs markedly from the solution using the measured contact wrenches. The benchmark only tries to minimize the norm of the joint torque, irrespective if this represents reality.

The remaining errors between the estimated and measured contact wrenches in Fig. 6 and resulting joint torques in Fig. 5 are partially due to modelling errors. It turns out that our 


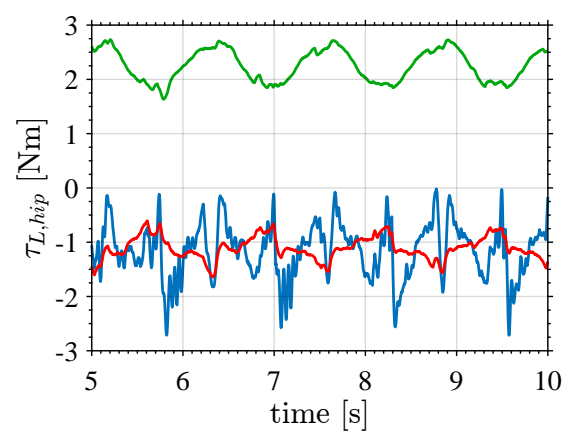

(a) $\tau_{L, \text { hip }}$

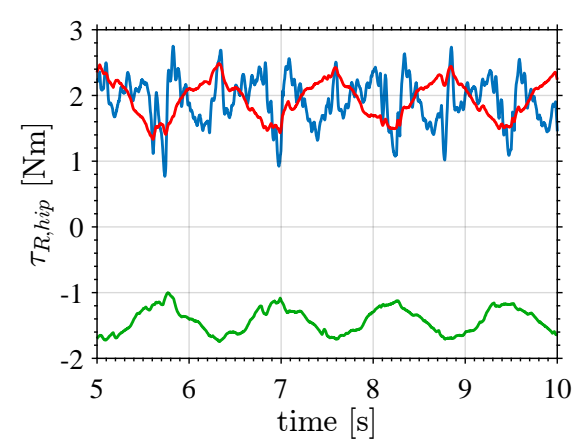

(d) $\tau_{R, \text { hip }}$

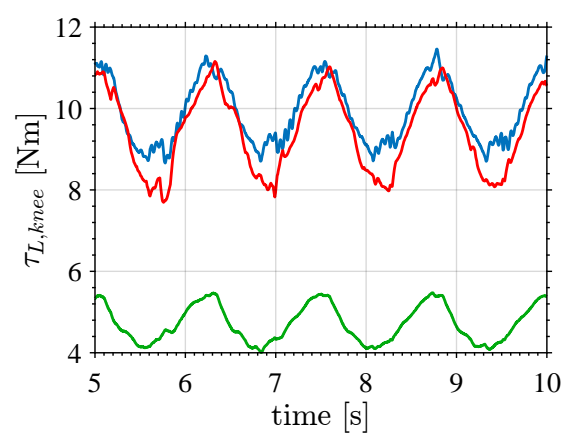

(b) $\tau_{L, k n e e}$

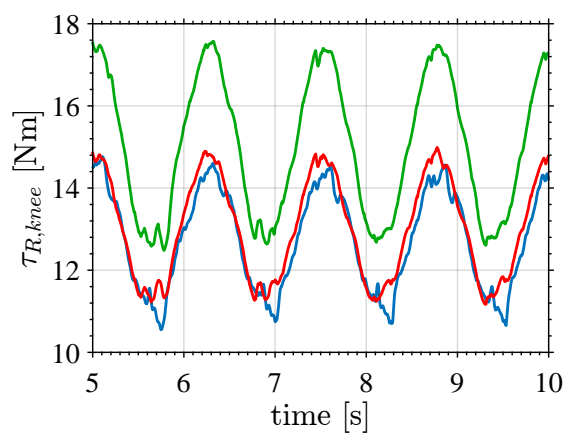

(e) $\tau_{R, \text { knee }}$

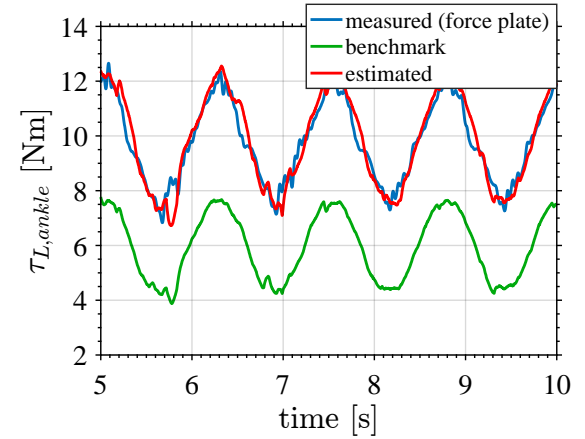

(c) $\tau_{L, \text { ankle }}$

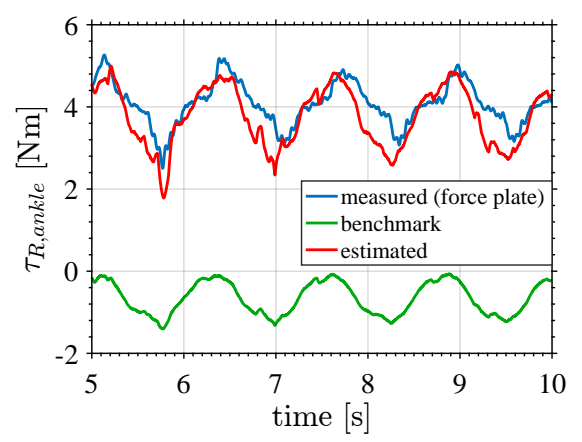

(f) $\tau_{R, \text { ankle }}$

Fig. 6. The exoskeleton joint torques using the estimated ground reaction wrenches (in red) are compared against the torques from the measured ones (in blue) and the torques from the benchmark method, which minimizes the norm of the actuation torques (in green). The exoskeleton joint torques of the hip, knee and ankle joint of both legs are all compared.

method is sufficiently accurate in view of practical applications, at least in a robotic system dominated by localized joint compliances. A linearised approximation of the stiffness already gives results close to the true measurements. This is indicated by the small increase in RMS errors between the estimation based on the non-linear and linearised stiffness (Table II). The good results of the linear approximation can be explained by the role of the stiffness in the estimation. The stiffness of the structure weights the torque/force contributions in the total energy. Hence, the absolute values of the stiffness are of no importance, but their mutual relative values are. This also explains why the simple modelling of the pelvis already gives reasonable contact wrench estimations compared to the measured ones. Although the pelvis complementary energy is a combination of mostly bending and shear energy, we model it on a macroscopic scale as a linear spring in two directions. This turns out to be sufficient to capture the dominant stiffness behaviour.

In case the dominating compliance cannot be localized at the joints as in SEAs, the compliance of the whole robot structure needs to be accounted for. The motors and gears of stiff robots (no SEAs) also display some compliance which can be modelled as very stiff SEAs. When the motors and gears have a large stiffness, the complementary energy of the stiff links cannot be ignored and plays an important role. The links are continuum elements with a non localized stiffness. To model their internal complementary energy, knowledge of the material properties and geometry of the robot links are required. Even for robots with dominating flexibility in a limited number parts, like SEAs, the accuracy of the result can be improved by modelling the complementary energy of every part of the robot. However, the gain in accuracy is expected to be limited for robots with flexible SEAs and stiff links. Hence, the added value should be outweighed against the added cost in time and effort.

The joint trajectories applied to validate the method are not directly related to a human motion. It resembles something between a squatting and a lunging motion, as both feet are not next to each other. The asymmetric position was chosen to make sure the wrench distribution would not be symmetric on both legs. The proposed method and methods from literature are only needed in the parts of gait when two feet are on the ground simultaneously. When only one foot of the walking robot or exoskeleton is on the ground, the inverse dynamics can be solved directly.

When using this method for exoskeletons for the lower limbs, it is only intended to be used for the contact forces of the exoskeleton itself. We aim for a controller which compensates the exoskeleton dynamics. The goal of this compensation is to make the exoskeleton move along with the person with interaction forces as small as possible. The proposed method to estimate ground reaction wrenches is needed to let the exoskeleton compensate itself. The proposed method can also be used in combination with assistance control methods which enhance or substitute human abilities. Assistance torques can be superimposed on the exoskeleton compensation torques. 
Our method is able to estimate the contact wrenches of a person in theory, but unable to do so in practice. First, a person is capable of changing the stiffness of his/her legs and joints by co-contracting muscles. Second, while modelling the skeletal model of a person is possible, modelling and measuring the stiffness is not feasible. If, however, the stiffness of each human joint is known, this method could be used to estimate the ground reaction wrenches of a person.

\section{CONCLUSION}

This paper presents a method based on complementary energy to estimate contact forces and moments for the inverse dynamics. The contact forces and moments can no longer be determined uniquely when the number of independent contact forces and moments exceeds the number of total system equations. Additional relations or equations are needed. The proposed method takes the stiffness of the robot into account by formulating the complementary energy of the robot. The minimization of this complementary energy provides the additional equations to solve the contact forces and moments.

The proposed method to estimate contact wrenches distinguishes itself from the literature by giving solutions consistent with physical reality. Our method can be written in the same form as the general solution of Righetti et al. [8] for distributing contact forces with inverse dynamics control. However, our method provides a clear methodology, based on physical system parameters, such as stiffness, to compute the weight matrices. Hence, the weight matrices are directly generated by our method. Tuning of the weight parameters is replaced by modelling. Even if further tuning is needed, this tuning is carried out in terms of physical parameters, which is easier than tuning more abstract weights.

The method was validated on a bilateral lower-limb exoskeleton. The experiments show that our methodology is sufficiently robust against modelling errors in order to be applied in practical applications, at least in a robotic system dominated by localized joint compliances.

\section{ACKNOWLEDGMENT}

The Robotics Research Group is a university core lab of Flanders Make. Jonas Vantilt is a research fellow of the Flemish agency for Innovation by Science and Technology (IWT-SBO 131381). The authors gratefully acknowledge the financial support by the agency Flanders Innovation \& Entrepreneurship (VLAIO) through a project grant (MIRAD, IWT-SBO 120057). The authors gratefully acknowledge the Multi-body and Dynamics group of Dirk Lefeber of the VUB for designing and building the exoskeleton used in the experiments. The authors also thank Amber K. B. D. Bruijnes for assisting in the measurements and Vicon labelling of the recorded data.

\section{REFERENCES}

[1] C. A. Klein, K. W. Olson, and D. R. Pugh, "Use of force and attitude sensors for locomotion of a legged vehicle over irregular terrain," International Journal of Robotics Research, vol. 2, no. 2, pp. 3-17, 1983.
[2] K. Nishiwaki, Y. Murakami, S. Kagami, Y. Kuniyoshi, M. Inaba, and H. Inoue, "A six-axis force sensor with parallel support mechanism to measure the ground reaction force of humanoid robot," in Proceedings 2002 IEEE International Conference on Robotics and Automation, vol. 3, May 2002, pp. 2277-2282.

[3] F. Aghili, "A unified approach for inverse and direct dynamics of constrained multibody systems based on linear projection operator: applications to control and simulation," IEEE Transactions on Robotics, vol. 21 , no. 5 , pp. 834-849, Oct 2005 .

[4] J. Park and O. Khatib, "Contact consistent control framework," in Proceedings of the 2006 IEEE International Conference on Robotics and Automation, Orlando, Florida, USA, May 2006, pp. 1963-1969.

[5] L. Sentis, J. Park, and O. Khatib, "Compliant control of multicontact and center-of-mass behaviors in humanoid robots," IEEE Transactions on Robotics, vol. 26, no. 3, pp. 483-501, Jun. 2010.

[6] M. Mistry, J. Buchli, and S. Schaal, "Inverse dynamics control of floating base systems using orthogonal decomposition," in 2010 IEEE International Conference on Robotics and Automation, May 2010, pp. 3406-3412.

[7] L. Righetti, J. Buchli, M. Mistry, and S. Schaal, "Control of legged robots with optimal distribution of contact forces." IEEE, Oct 2011, pp. $318-324$.

[8] L. Righetti, J. Buchli, M. Mistry, M. Kalakrishnan, and S. Schaal, "Optimal distribution of contact forces with inverse-dynamics control," The International Journal of Robotics Research, vol. 32, no. 3, pp. 280298, Mar. 2013.

[9] A. Del Prete, N. Mansard, F. Nori, G. Metta, and L. Natale, "Partial force control of constrained floating-base robots," in 2014 IEEE/RSJ International Conference on Intelligent Robots and Systems, Chicago, IL, US, Sep 2014, pp. 3227-3232.

[10] A. Herzog, L. Righetti, F. Grimminger, P. Pastor, and S. Schaal, "Balancing experiments on a torque-controlled humanoid with hierarchical inverse dynamics." IEEE, Sep 2014, pp. 981-988.

[11] B. Henze, C. Ott, and M. A. Roa, "Posture and balance control for humanoid robots in multi-contact scenarios based on Model Predictive Control," in IEEE/RSJ International Conference on Intelligent Robots and Systems, Chicago, IL, USA, Sept 2014, pp. 3253-3258.

[12] E. Sariyildiz and K. Ohnishi, "On the explicit robust force control via disturbance observer," IEEE Transactions on Industrial Electronics, vol. 62, no. 3, pp. 1581-1589, Mar 2015.

[13] A. Mohammadi, M. Tavakoli, H. Marquez, and F. Hashemzadeh, "Nonlinear disturbance observer design for robotic manipulators," Control Engineering Practice, vol. 21, no. 3, pp. 253 - 267, 2013.

[14] M. Saadatzi, C. D. Long, and O. Celik, "Comparison of humanrobot interaction torque estimation methods in a wrist rehabilitation exoskeleton," Journal of Intelligent \& Robotic Systems, Feb 2018.

[15] B. Ugurlu, M. Nishimura, K. Hyodo, K. Michihiro, and N. Tatsuo, "Proof of concept for robot-aided upper limb rehabilitation using disturbance observers," IEEE Transactions on Human-Machine Systems, vol. 45, no. 1, pp. 110-118, Feb 2015.

[16] O. Bauchau and J. Craig, Energy Methods. Springer New York, 2009, ch. 10 , pp. $493-581$.

[17] J. N. Reddy, Energy Principles and Variational Methods in Applied Mechanics. John Wiley \& Sons Ltd., 2017.

[18] F. Roy, "Modelling rigid body systems," in Rigid Body Dynamics Algorithms, F. Roy, Ed. Springer, 2008, ch. 4, pp. 65-87.

[19] C. Lanczos, The variational principles of mechanics. Courier Corporation, 2012.

[20] K. Junius, B. Brackx, V. Grosu, H. Cuypers, J. Geeroms, M. Moltedo, B. Vanderborght, and D. Lefeber, "Mechatronic design of a sit-to-stance exoskeleton," in 2014 IEEE RAS \& EMBS International Conference on Biomedical Robotics and Biomechatronics (BioRob), São Paulo, Brazil, August 12-15 2014, pp. 945-950.

[21] A. De Luca and W. Book, "Robots with flexible elements," in Springer Handbook of Robotics, B. Siciliano and O. Khatib, Eds. SpringerVerlag, Berlin, Heidelberg, 2008, ch. A.13, pp. 321-344.

[22] J. Vantilt, E. Aertbeliën, F. De Groote, and J. De Schutter, "Optimal excitation and identification of the dynamic model of robotic systems with compliant actuators," in 2015 IEEE International Conference on Robotics and Automation, Seattle, Washington, USA, May. 2015, pp. 2117-2124. 